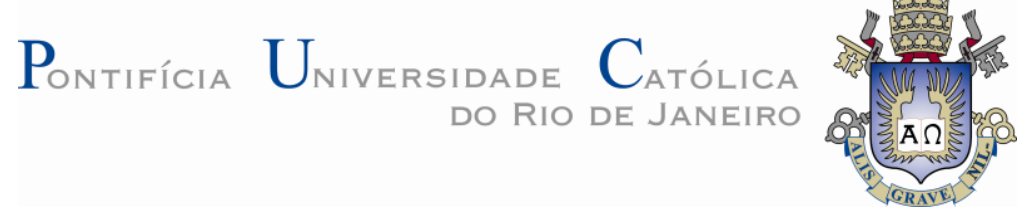

Felipe Siqueira Schouchana

\title{
Decisão ótima em Swaps de energia elétrica no Brasil utilizando a medida Omega
}

\section{Dissertação de Mestrado (Opção profissional)}

Dissertação apresentada como requisito parcial para a obtenção do grau de Mestre pelo Programa de Pósgraduação em Administração de Empresas do Departamento de Administração da PUC-Rio.

Orientador: Prof. Leonardo Lima Gomes

Rio de Janeiro

Setembro de 2010 


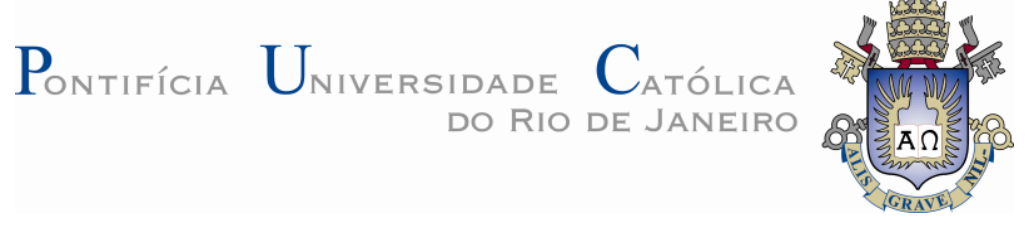

Felipe Siqueira Schouchana

\section{Decisão ótima em Swaps de energia elétrica no Brasil utilizando a medida Omega}

Dissertação apresentada como requisito parcial para obtenção do grau de Mestre pelo Programa de Pósgraduação em Administração de Empresas da PUC-Rio. Aprovada pela Comissão Examinadora abaixo assinada.

Prof. Marcelo Cabus KIotzle Departamento de Administração - PUC-Rio

Prof. Paulo Henrique Soto Costa UERJ

Prof ${ }^{a}$. Mônica Herz Vice-Decana de Pós-Graduação do CCS

Rio de Janeiro, 9 de setembro de 2010 
Todos os direitos reservados. É proibida a reprodução total ou parcial do trabalho sem autorização da universidade, do autor e do orientador.

Felipe Siqueira Schouchana

Graduou-se em Administração de Empresas pela Pontifícia Universidade Católica do Rio de Janeiro (PUC-Rio) em 2003. Trabalhou em grandes empresas como CSN Companhia Siderúrgica Nacional, Endesa Brasil, Abengoa e Telemar, tendo atuado no setor elétrico por 5 anos. Atualmente trabalha na Praxair/White Martins. Especializouse em Management pelo IAG - PUC-Rio em 2008.

Ficha Catalográfica

\section{Schouchana, Felipe Siqueira}

Decisão ótima em swaps de energia elétrica no Brasil utilizando a medida Ômega / Felipe Siqueira Schouchana ; orientador: Leonardo Lima Gomes. - 2010.

69 f. : il. (color.) ; $30 \mathrm{~cm}$

Dissertação (mestrado)-Pontifícia Universidade Católica do Rio de Janeiro, Departamento de Administração, 2010.

Inclui bibliografia 


\section{Agradecimentos}

Em primeiro lugar, gostaria de agradecer aos meus pais, Cidda Siqueira e Edwin Schouchana que, mesmo a distância, sempre estiveram presentes me dando força em todos os momentos.

À minha namorada Lara Grant por todo o incentivo dado.

Ao Professor Leonardo Lima Gomes, pela orientação e estimulo para a realização deste trabalho.

À Marta Dalbem, pela ajuda na revisão deste trabalho. 


\section{Resumo}

Schouchana, Felipe Siqueira; Gomes, Leonardo Lima. Decisão Ótima em Swaps de Energia Elétrica no Brasil Utilizando a Medida Omega. Rio de Janeiro, 2010. 69 p. Dissertação de Mestrado - Departamento de Administração. Pontifícia Universidade Católica do Rio de Janeiro.

O setor elétrico brasileiro passou por reestruturações que tiveram início no final da década de 90 , objetivando aumentar a eficiência dos agentes e do mercado como um todo. Neste processo, foi criado o ACR - Ambiente de Contratação Regulada e o ACL - Ambiente de Contratação Livre. Contratações no ACL podem ser a preços fixos e pré-determinados pelas partes ou, ainda, variáveis conforme o PLD - Preço da Liquidação das Diferenças. O PLD pode apresentar comportamento volátil, se tornando um fator de alto risco para os agentes do setor elétrico. A matriz elétrica é divida em quatro submercados: Norte, Sul, Nordeste e Sudeste/Centro-Oeste, sendo que cada um apresenta PLDs distintos em função de restrições na malha de transmissão entre outros fatores. No entanto, é comum que as comercializadoras façam Swap de energia elétrica, que consiste em comercializar energia entre diferentes submercados, para lucrar com as diferenças de preços. A gestão do risco inerente a essas operações geralmente é realizada pelas próprias empresas, as quais utilizam modelos financeiros para otimização de portfólio de contratos levando em conta métricas de risco. Assim, a comercializadora irá contabilizar um lucro ou prejuízo dependendo dos preços de cada submercado. Nesse contexto, o presente estudo oferece um modelo de decisão ótima em Swaps de energia elétrica utilizando como balizador de desempenho a medida Ômega com restrição de valor em risco (VaR - Value at Risk) de forma a auxiliar comercializadores a maximizarem seus lucros, minimizando riscos, por meio de operações casadas.

Palavras-chave: Swap de Energia, Medida Ômega; Energia Elétrica; PLD 


\section{Abstract}

Schouchana, Felipe Siqueira; Gomes, Leonardo Lima. Optimal Decision in Electric Power Swaps in Brazil Using the Omega Measurement. Rio de Janeiro, 2010. 69 p. Dissertation - Departamento de Administração. Pontifícia Universidade Católica do Rio de Janeiro.

The Brazilian electric sector underwent a major restructuring that began at the end of the 1990s, which aimed to increase the efficiency of the agents and the market as a whole. This process created the Regulated Contracting Environment (ACR) where the generators sell their energy through government-organized auctions, and the Free Contracting Environment (ACL) which enables deals between producers and consumers whose demand exceeds $3 \mathrm{MW}$, directly or via brokers. Purchases in the ACL may be at fixed or previously-set prices, or even at floating prices based on the Spot Price (PLD). The PLD may be volatile, becoming a high risk factor for the electric sector agents. Energy supply is divided into four sub-markets: North, South, Northeast and Southeast/Midwest, and each presents a distinct PLD due to restrictions on the transmission lines and other factors. However, it is common for agents to trade in electric power swaps, which means trading energy among different sub-markets and profiting from price differences. The management of risk inherent in such operations is usually performed by the companies themselves, which use financial models to optimize contract portfolios, taking into account risk metrics. Thus, the trader will book a profit or loss depending on the prices in each sub-market. Against this backdrop, this study offers a model of optimal decision in Electric Power Swaps (buying and selling the same amount of energy) using the Omega Measurement as a performance indicator, restricted by value at risk $(\operatorname{VaR})$ to help brokers maximize their profit while minimizing risks.

\section{Keywords:}

Energy Swap, Omega Measurement; Electric Power; PLD 


\section{Sumário}

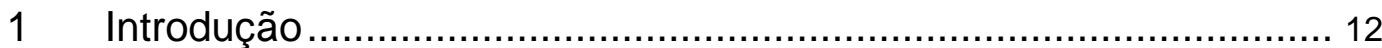

2 Características do mercado brasileiro de energia elétrica .............. 19

2.1 Modelos de geração e formação do preço de energia elétrica ........ 19

2.2 Operação de um sistema hidrotérmico ...................................... 22

2.3 Modicidade tarifária, revisão tarifária e leilões de energia ............... 23

2.4 Estratégias de comercialização de energia elétrica ....................... 25

2.4.1Liquidação de $100 \%$ dos contratos de venda no mercado de curto

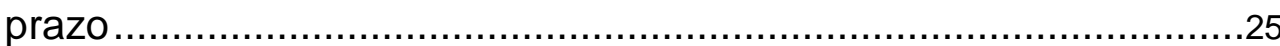

2.4.2 Liquidação parcial dos contratos de venda no mercado de curto

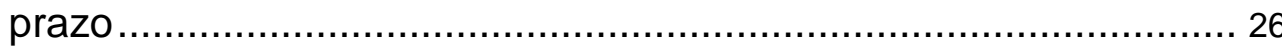

2.4.3Swap de energia elétrica - compra e venda entre diferentes

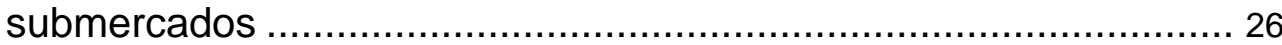

2.5 Dinâmica da decisão da comercialização de energia elétrica.......... 28

3 Revisão da literatura e conceituação da medida ômega $(\Omega)$............ 30

3.1 Risco e retorno de investimentos.............................................. 30

3.2 Teorias de escolha de carteiras ................................................ 31

3.2.10 modelo de Markovitz ...................................................... 31

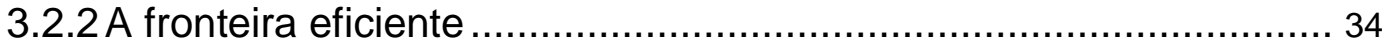

3.2.30 índice de Sharpe .................................................................... 36

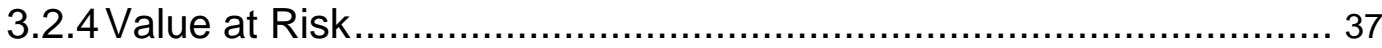

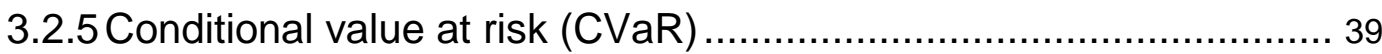

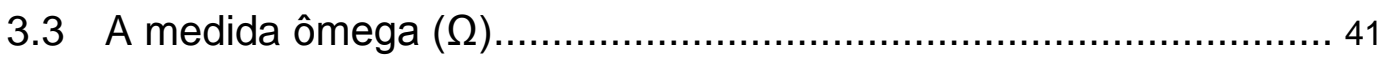

3.3.1 Definição e cálculo da medida ômega ....................................... 42

3.3.2 Representação alternativa da medida ômega ............................. 47

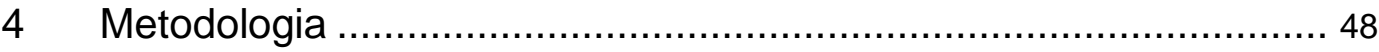

4.1 Modelagem - construindo Swap ótimo de contratos de energia elétrica ................................................................................ 48

4.1.1 Construção do modelo financeiro ............................................. 49

4.1.2 Equação de resultado ......................................................... 52 


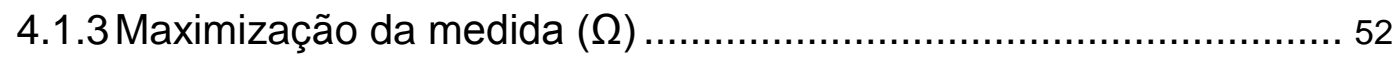

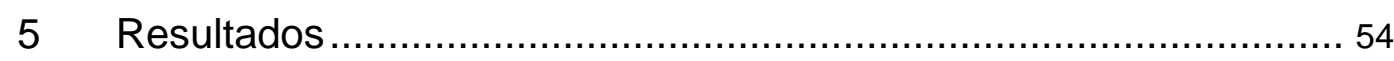

5.1 Simulação dos PLDs......................................................... 54

5.2 Etapas das simulações de contratação de energia entre o submercado SE e os demais submercados............................... 57

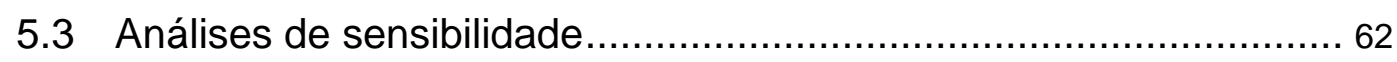

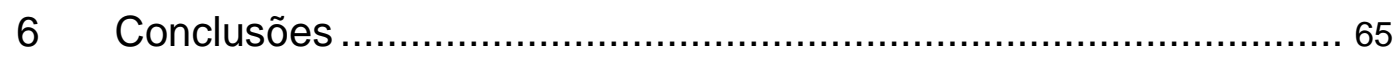

6.1 Sugestões para trabalhos futuros ....................................... 66

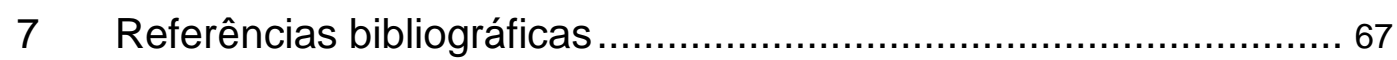




\section{Lista deTabelas}

Tabela 1: Tabela comparativa do sistema elétrico brasileiro, antes e depois da reforma ..................................................................... 14

Tabela 2: Tabela de retorno e ganhos (ou perdas) em relação à meta $\mathrm{L}=3,5$ 43

Tabela 3: retorno, ganho ou perda em relação à meta, probabilidade de ocorrência.............................................................................................. 44

Tabela 4: Tabela com as siglas utilizadas ........................................ 49 Tabela 5: Tabela de compra SE e venda nos demais submercados

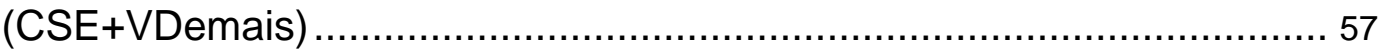

Tabela 6: Tabela de venda SE e compra nos demais submercados

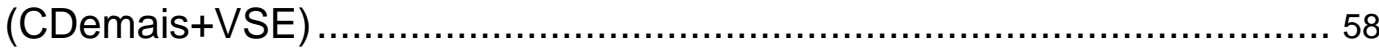

Tabela 7: Tabela de compra SE e venda nas demais regiões ................ 59

Tabela 8: Tabela de compra demais regiões e venda SE ..................... 59

Tabela 9: Tabela com combinações em duplas ...................................6 60

Tabela 10: Tabela com combinações entre as três opções de maior medida $\Omega$ 61 


\section{Lista de Figuras}

Figura 1: Mercado de curto prazo

Figura 2: Cenário de utilização das reservas de energia hidráulica e suas conseqüências 22

Figura 3: Evolução dos custos imediato e futuro, e decisão ótima 23

Figura 4: Transação entre submercados 27

Figura 5: Árvore de decisão para comercialização de energia elétrica. 28

Figura 6: Fronteira eficiente 35

Figura 7: Intervalo de confiança 38

Figura 8: llustração do CVaR e do VaR 40

Figura 9: Exemplo de distribuição de probabilidade dos retornos 42

Figura 10: Exemplo de perda e ganho esperado 44

Figura 11: llustração das parcelas ganho esperado e perda esperada em uma distribuição contínua 46

Figura 12: Numerador $E C(L)$ e denominador $E S(L)$ do cálculo da medida $\Omega \quad 47$

Figura 13: Histograma distribuição PLDs Sudeste/Centro-Oeste - média anual

Figura 14: Distribuição dos PLDs dos quatro submercados para 2011 média anual 55

Figura 15: Histogramas de diferenças de preços entre submercados $\quad 56$

Figura 16: Simulação CSE-VNE 62

Figura 17: Simulação CS-VSE 63

Figura 18: Simulação CSE-VN 63 


\section{Lista de Quadros}

Quadro1: Primeira simulação de contratos $\quad 50$

Quadro 2: Segunda simulação de contratos 50

Quadro 3: Teste de portifólio - (i) 51

Quadro 4: Teste de portifólio - (ii) 51 